\title{
Discovery of Novel Human Aquaporin-1 Blockers
}

\author{
Daniel Seeliger, ${ }^{\dagger, \perp}$ Cinta Zapater, ${ }^{\ddagger} \perp$ Dawid Krenc, ${ }^{\text {II }}$ Rose Haddoub, ${ }^{\S}$ Sabine Flitsch, ${ }^{\S}$ Eric Beitz, ${ }^{\text {II }}$ \\ Joan Cerdà, $* * *$ and Bert L. de Groot* ${ }^{\dagger}$ \\ ${ }^{\dagger}$ Computational Biomolecular Dynamics Group, Max-Planck-Institute for Biophysical Chemistry, 37077 Göttingen, Germany \\ ${ }^{\ddagger}$ Institut de Recerca i Tecnologia Agroalimentàries (IRTA)-Institut de Ciències del Mar, Consejo Superior de Investigaciones \\ Científicas (CSIC), 08003 Barcelona, Spain \\ "Department of Pharmaceutical and Medicinal Chemistry, University of Kiel, Gutenbergstr. 76, 24118 Kiel, Germany \\ ${ }^{\S}$ School of Chemistry and Manchester Interdisciplinary Biocentre (MIB), The University of Manchester, Manchester M1 7DN, U.K.
}

\section{Supporting Information}

ABSTRACT: Human aquaporin-1 (hAQP1) is a water channel found in many tissues and potentially involved in several human pathologies. Selective inhibitors of hAQP1 are discussed as novel treatment opportunities for glaucoma, brain edema, inflammatory pain, and certain types of cancer. However, only very few potent and chemically attractive blockers have been reported to date. In this study we present three novel hAQP1 blockers that have been identified by virtual screening and inhibit water flux through hAQP1 in Xenopus laevis oocyte swelling assays at low micromolar concentrations. The newly discovered compounds display no chemical similarity to hitherto known hAQP1 blockers and bind at the extracellular entrance of the channel, close to the ar/R selectivity filter. Futhermore, mutagenesis studies showed that Lys36, which is not conserved among the hAQP family, is crucially involved in binding and renders the discovered compounds suitable as leads for the development of selective hAQP1 inhibitors.

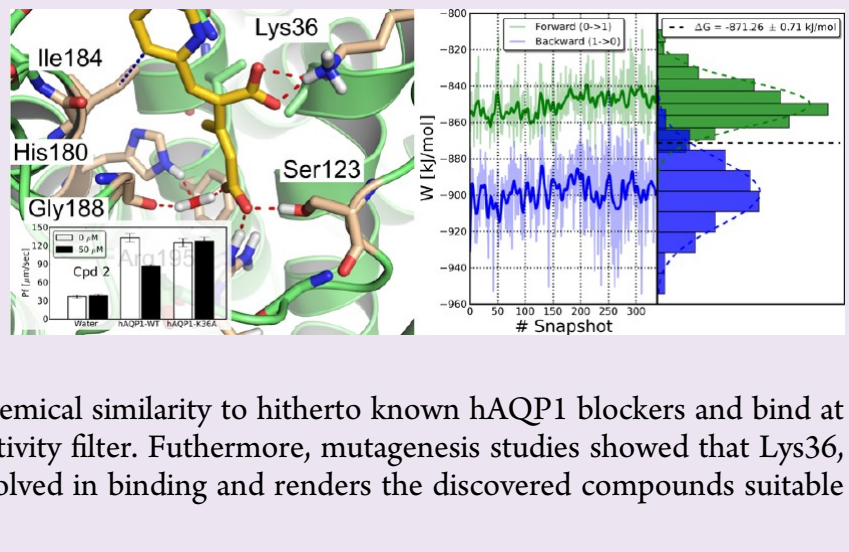

is proposed to facilitate cerebrospinal fluid formation. ${ }^{12,13}$ It has been shown that intraventricular pressure was reduced by $50 \%$ in mice lacking AQP1, suggesting that hAQP1 is a major contributor to the production and removal of brain fluid volume homeostasis. ${ }^{14}$ An increased expression of hAQP1 has been reported in Creutzfeld-Jakob disease, indicating an involvement in prion diseases. ${ }^{15}$ The particular roles of different AQPs in the brain are not yet well understood, and it remains speculative whether AQP-blockers are suitable for the treatment of brain-related diseases. However, having efficient blockers as tool compounds at hand would certainly facilitate novel research opportunities and contribute to the understanding of AQP function in the brain. Similar prospects apply accordingly for the role of AQPs in the treatment of cancer. It has been shown that hAQP1 is upregulated in certain cancer types, e.g., glioblastoma, and that the level of aquaporin expression correlates with the aggressiveness of the tumor. ${ }^{16,17}$ $\mathrm{hAQP1}$ is essential for tumor angiogenesis and responsible for an acceleration of endothelial cell migration and, hence, with tumor spread. ${ }^{18-20}$ Recent findings furthermore suggest hAPQ1 to be involved in the perception of inflammatory pain in dorsal root ganglions through interaction with the sodium channel $\mathrm{Na}_{\nu} 1.8 .^{21}$ Modulation of hAQP1 therefore

Received: April 3, 2012

Accepted: October 31, 2012

Published: October 31, 2012 


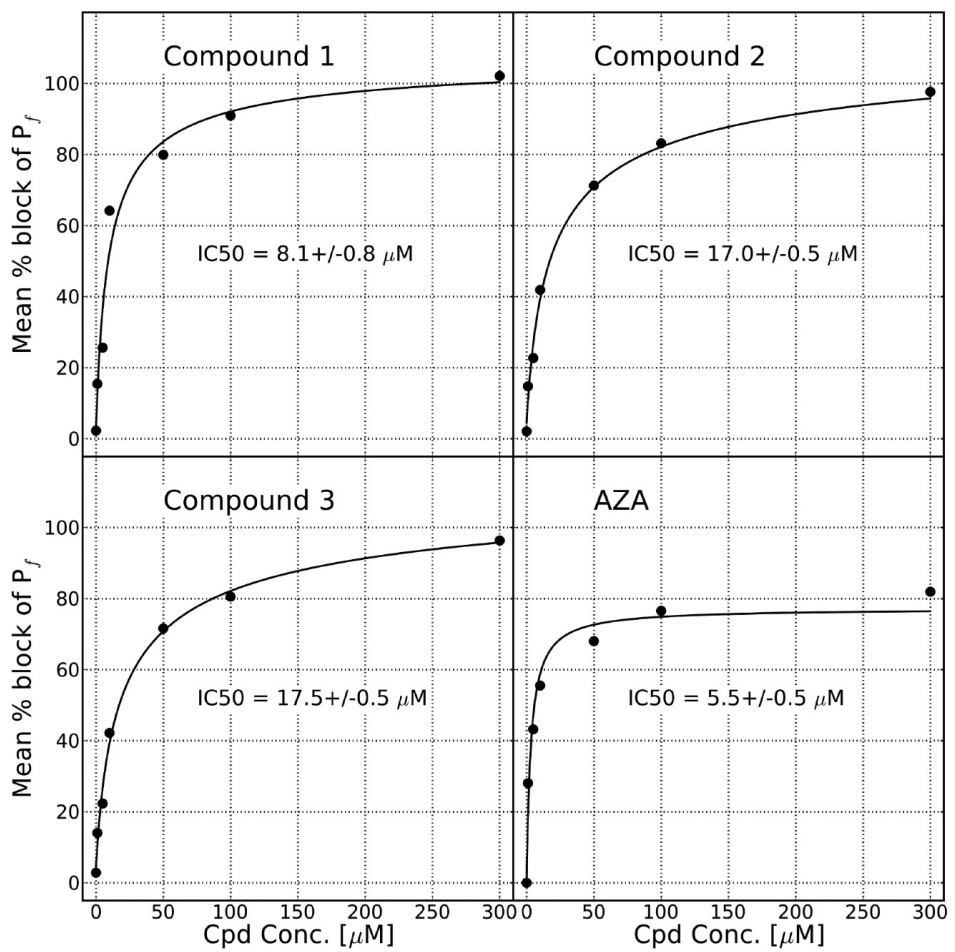<smiles>O=C(O)/C=C/c1ccccc1/C=C/C(=O)O</smiles><smiles>[2H]/C(=C\c1ccc2ccccc2n1)C(=CC(=O)O)C(=O)O</smiles><smiles>CC(=S)Nc1ccc2nsnc2c1</smiles><smiles>CCc1ccc2c(c1)OCO2</smiles><smiles>CC(=O)Nc1nnc(S(N)(=O)=O)s1</smiles>

Figure 1. Inhibition of water permeability through hAQP1-WT expressed in Xenopus leavis oocytes by three novel compounds (cpd1, cpd2, cpd3) and acetazolamide (AZA). Data (mean \pm SEM, $n=30$ oocytes) are the percentage of block of $P_{\mathrm{f}}$ compiled from three independent experiments performed on differend batches of oocytes. The half maximal inhibitory concentration $\left(\mathrm{IC}_{50}\right) \pm \mathrm{SEM}$, calculated from the three experiments, is indicated for each compound.

might influence membrane $\mathrm{Na}^{+}$currents and, hence, could be an attractive target for the treatment of inflammatory pain disorders.

Because of the numerous important roles of AQPs in physiology and their possible utilization as targets for future medication, the development of potent and selective modulators, in particular inhibitors, is highly desired to foster future research in in vitro and in vivo systems beyond knockout mice. $^{22,23}$ However, despite the attractiveness of hAQP1 as drug target no potent and selective inhibitors could be identified yet.

The first chemical that has been found to inhibit water flux in AQPs was mercuric chloride, which covalently binds to cysteine 189 at the extracellular entrance of the pore. ${ }^{24}$ Later it was found that tetraethylammonium (TEA), a blocker of voltagegated potassium channels, calcium-dependent potassium channels, and the nicotinic acetylcholine receptor, also affects the activity of AQPs. ${ }^{25-27}$ More recently, silver and gold have also been shown to inhibit AQPs. ${ }^{28}$ However, these metals are toxic and, like mercuric chloride, bind covalently to Cys189. Ma et al. $^{29}$ showed that acetazolamide, a carbonic anhydrase inhibitor, is also an efficient blocker of hAQP1. Recently, Migliati et al. ${ }^{30}$ reported the discovery of AqB013, a bumetanide derivative that inhibits water flux in swelling assays of hAQP1 and hAQP4. The $\mathrm{IC}_{50}$ of AqB013 is $\sim 20 \mu \mathrm{M}$ for both hAQP1 and hAQP4. In contrast to the previously known inhibitors, AqB013 binds to an intracellular binding site that was confirmed by mutagenesis studies.

A major obstacle for the identification of AQP inhibitors is the difficulty to establish robust and reliable assays with AQPdependent reduction of water flux as read-out. The AQPblocking effect of TEA and acetazolamide for instance has been shown in AQP-expressing MDCK cells and Xenopus oocytes. However, the blocking effect could not be reproduced with erythrocytes and hAQP1-expressing epithelial cells. ${ }^{31}$

In this study we present a set of molecules that inhibit water flux across hAQP1. The novel compounds were identified by molecular docking against the extracellular part of hAQP1 and experimentally tested in a Xenopus oocyte swelling assay. Subsequent molecular dynamics simulations suggest a binding mode that strongly involves the ar/ $\mathrm{R}$ selectivity filter and Lys36, a residue that is not conserved among the hAQP familiy. The proposed binding mode, which is distinct from previously known hAQP1 inhibitors, was confirmed by mutagenesis studies and renders the presented compounds as attractive starting points for the development of selective hAQP1 inhibitors.

\section{RESULTS AND DISCUSSION}

hAQP1 Inhibition in Xenopus oocytes. Analysis of the top hit list obtained from virtual screening revealed a rather simple pharmacophore as dominating feature, a carboxylic group pointing toward Arg195 and an aromatic moiety located in proximity to different hydrophobic residues at the entrance of the water channel. After visual inspection of the binding poses and evalutation of vendor availability 14 compounds from the top 50 list were purchased and experimentally tested in an hAQP1-cRNA injected Xenopus laevis oocyte assay. Three compounds (see Figure 1) showed a significant reduction of osmotic swelling, and $\mathrm{IC}_{50}$ determination resulted in 8.1, 17.0, and $17.5 \mu \mathrm{M}$ inhibition of water flux across hAQP1 (Figure 1), which is comparable to the inhibition of acetolazetamide, which showed an $\mathrm{IC}_{50}$ of $5.5 \mu \mathrm{M}$ in our experimental setup. (A table of all tested compounds is shown in the Supporting Materials). 
Analysis of Binding Modes. It is remarkable that two of the novel compounds are negatively charged whereas the hitherto known extracellular inhibitors are either neutral or positively charged. Binding of quartenary ammonium compounds such as tetraethylammonium (TEA) to hAQP1 has been studied previously by docking and molecular dynamics simulations $^{32}$ and suggested a binding mode that strongly involves the A-loop of the neighboring monomer. The binding modes obtained from docking of the novel compounds, however, suggest a less dominant role of the neighboring Aloop but rather a strong involvement of the ar/R selectivity filter in ligand binding. The hAQP1 selectivity filter consists of Arg195, Phe56, and His180 and forms the narrowest part of the water channel. Small-molecule inhibitors binding to this bottleneck of the water channel are therefore likely to efficiently reduce the water flux across the channel.

In order to confirm the proposed binding modes from molecular docking, we performed molecular dynamics simulations starting from the docked conformations. Due to the short, sub-microsecond simulation time scales, stable binding modes observed in MD trajectories do not necessarily confirm a specific binding mode, but energetically unfavorable conformations usually result in either a reorientation of the ligand or, more likely, in the dissociation of the complex over the course of the simulation. Although dissociation and association rates differ substantially between different protein/ ligand complexes, they are usually too slow to be observed within the time scales accessible by MD simulations. A stable $\mathrm{MD}$ trajectory can therefore be regarded as a minimum requirement for a stable binding mode.

The simulations showed that compounds $\mathbf{1}$ and $\mathbf{2}$ remained stably bound in several simulations on time scales of several hundred nanoseconds, whereas compound 3 turned out to be very flexible in most simulations and in one simulation even adopted an "inverted" conformation as compared to the initial docking pose (see Figure 2).

Compounds 1 and $\mathbf{2}$ share greater similarity according to Tanimoto coefficients ${ }^{33,34}$ than each of them compared to compound 3. Both have two carboxylic groups and aromatic moieties, and the predicted binding modes in both cases show a carboxylic group pointing toward Arg195 (Figure 3C and D), which results in a disruption of the transmembrane water file (see Figure 3F).

Since the experimental data revealed a decrease of the water permeation rate, we also assessed the inhibitory effect of the compounds on the water permeation in the simulations. Figure 4 shows the permeation rates calculated for the three free pores of the hAQP1 tetramer and the permeation rate for the ligandbound pore. As can be seen, with bound ligand, water flux across the membrane decreases by approximately $75 \%$ for all three compounds.

Binding Mode Confirmation with a hAQP1-K36A Mutant. While the transmembrane region and the selectivity filter of hAQP1 are conformationally relatively rigid, the extracellular loops display a high degree of flexibility. In all simulations, Lys36 was observed to interact strongly with either the aromatic moieties of compounds $\mathbf{1}$ and $\mathbf{3}$ or with one of the acidic groups of compound 2 . This interaction is particularly interesting as Lys36 is unique among the human AQP familiy (see Figure 3E) and therefore a potential path toward selective hAQP1 inhibition. In order to quantify the contribution of Lys36 to the binding affinity we carried out free energy calculations for the K36A mutation for compounds 1 and 2

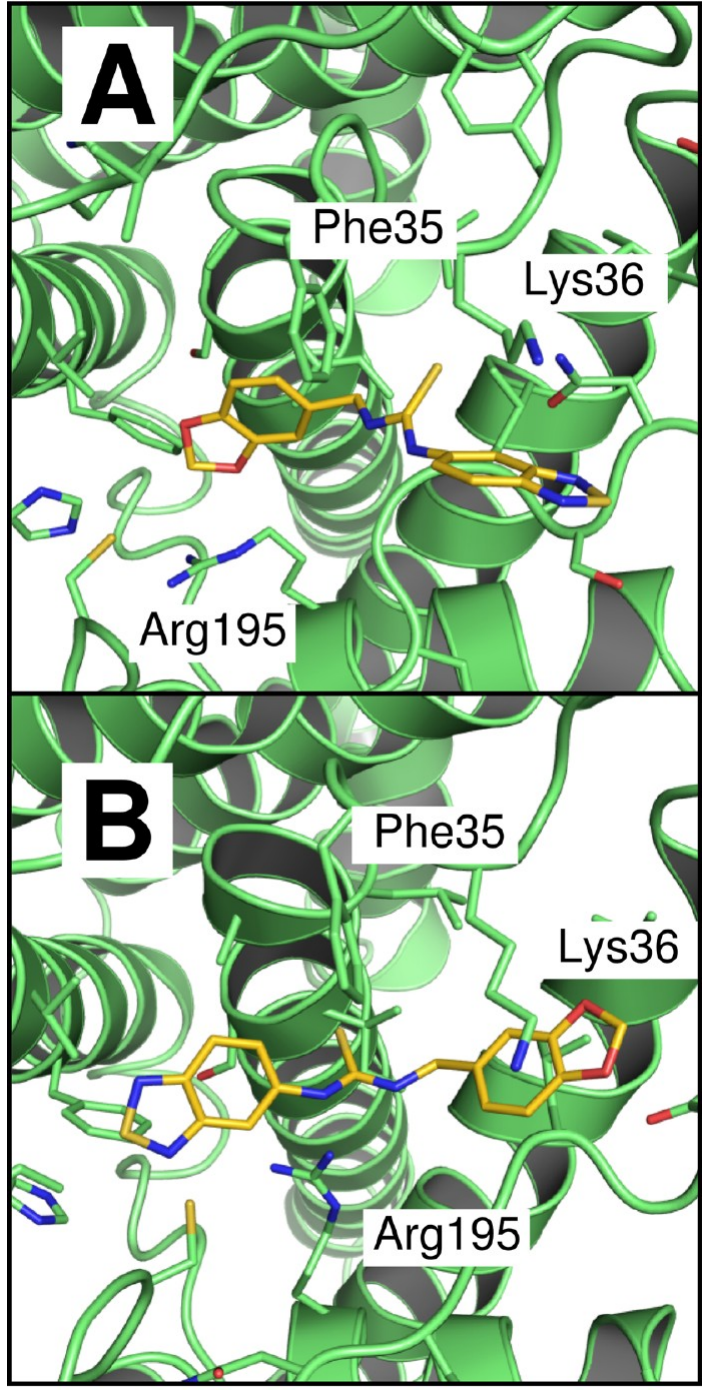

Figure 2. MD simulations of compound 3. (A) Binding mode at the beginning of the simulation. (B) Binding mode after $100 \mathrm{~ns}$.

using a previously established simulation protocol. ${ }^{35,36}$ The calculations (see Supporting Material) predict that the hAQP1K36A mutation results in a drop of the $K_{\mathrm{D}}$ by $2-3$ orders of magnitude for compounds $\mathbf{1}$ and $\mathbf{2}$ compared to wild type hAQP1 and hence would serve as a strong validation of the proposed binding mode. We therefore generated the hAQP1K36A mutant and repeated the permeation experiments for the three novel compounds and for acetazolamide. As can be seen in Figure 5, the inhibitory effect of all three compounds disappears, while AZA blocks water flux across hAQP1-K36A with efficacy comparable to that of WT-hAQP1. These results clearly show that Lys36 plays, as predicted by the simulations, a pivotal role for binding of the novel compounds and strongly support the proposed binding site at the extracellular entrance, which is distinct from previously reported binding sites. It furthermore revealed that compound 3 , for which no clear binding mode could be predicted from docking and $\mathrm{MD}$ simulations, also binds at the same binding site and interacts with Lys36.

Red Blood Cell Assay. Since previous studies on putative hAQP1 inhibitors revealed that compounds that inhibited water flux in Xenopus oocytes showed no effect in assay formats based on erythrocytes and hAQP1-expressing epithelial cells, ${ }^{31}$ we 


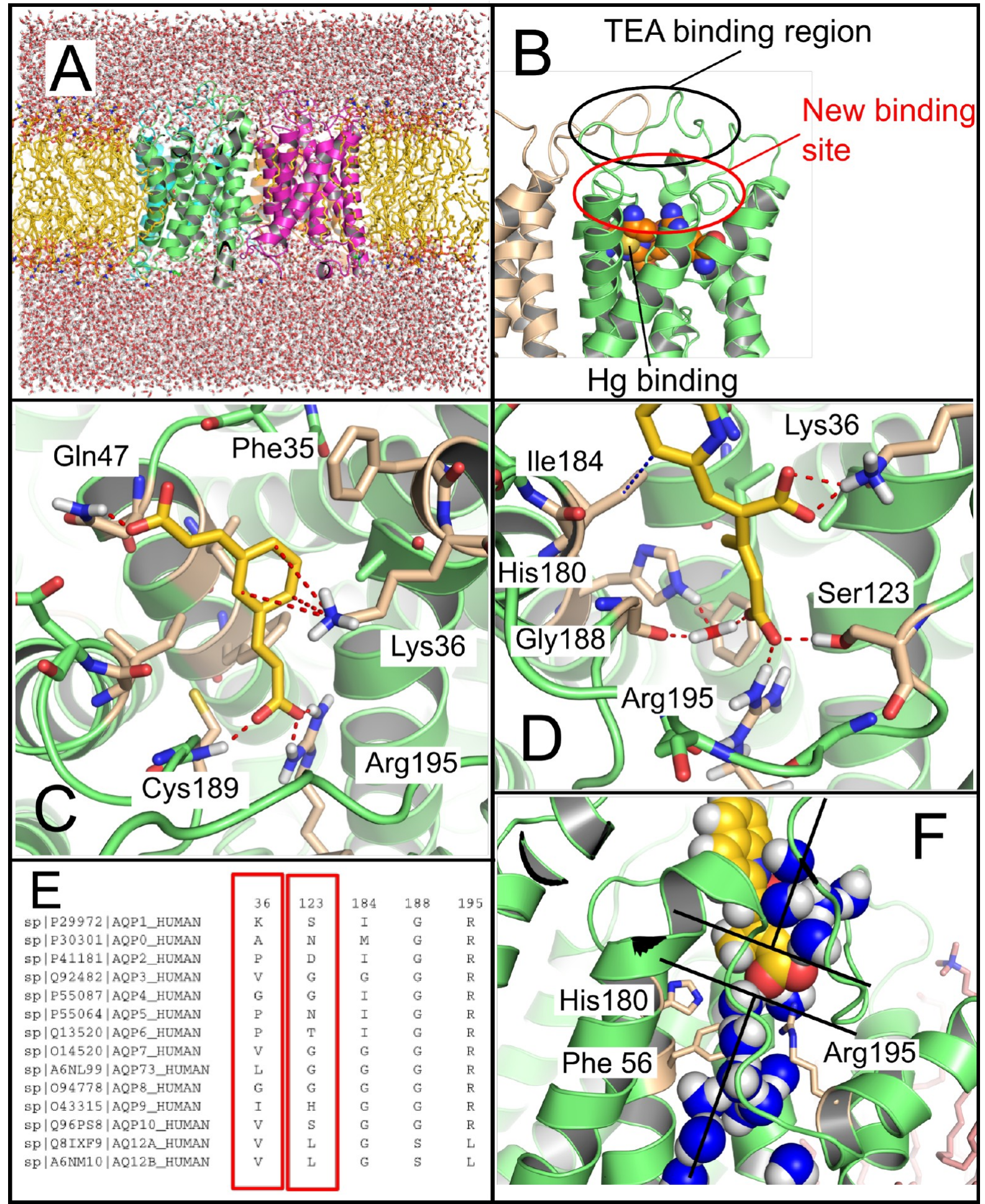

Figure 3. MD simulations and sequence comparison. (A) Simulation system of the hAQP1 tetramer embedded in a DOPC bilayer. (B) Location of the binding sites of TEA, Hg, and compounds 1 and 2. (C) MD snapshot of the binding site with compound 1. (D) MD snapshot of the binding site with compound 2. (E) Sequence alignment of strongly interacting residues in the binding site. Lys36 and Ser123 are not conserved among the hAQP family. (F) Ligand binding disrupts the water file across the channel.

tested the three compounds in a red blood cell assay. None of the molecules showed an inhibitory effect. Zhang and Verkman, who showed in their study that TEA does not lead to a significant effect of hAQP1 inhibition in these systems, raised the question of whether the apparent inhibition of water flux by TEA at high concentrations could be due to nonspecific effects such as the block of $\mathrm{K}^{+}$channels and consequent altered basal cell volume. Although such an effect cannot be ruled out for TEA, we would exclude such an effect for the compounds presented here. At least for compounds 1 and 2, which are negatively charged, blockage of a cation channel can be regarded as very unlikely. Furthermore, the fact that hAQP1$\mathrm{K} 36 \mathrm{~A}$ cannot be inhibited by the presented compounds is a 


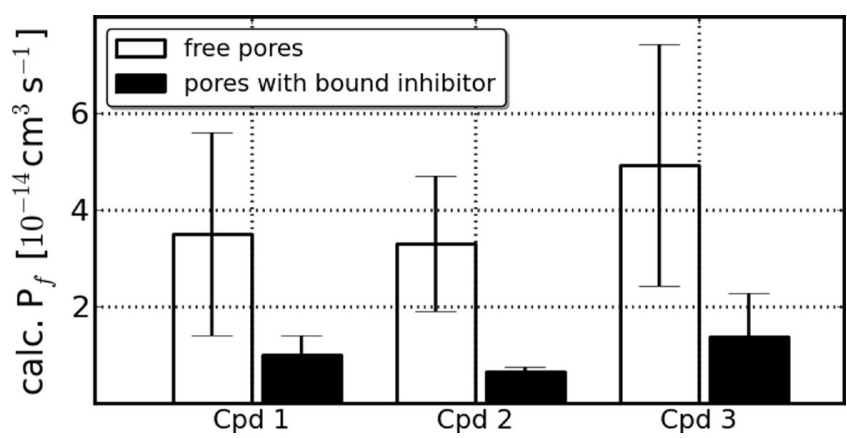

Figure 4. Calculated permeation rates. Permeation rates were calculated for the free pores and the pores with bound inhibitor. The reduction of water flux is $\sim 75 \%$ for all three compounds.

strong hint that the observed reduction of water flux is hAQP1dependent and not triggered by an indirect effect.

Nevertheless, the obvious discrepancy between results obtained from oocyte and erythrocyte assays remains and needs to be resolved. A possible explanation could be the lower density of hAQP1 in the membrane of injected oocytes and fewer channels render cells more sensitive to low-affinity binders at concentrations in the micro- or millimolar range. Hence, the obtained results are not necessarily contradictory, but the presented compounds might just not be potent enough to give a significant signal in red blood cell assays.

However, the fact that inibition of oocyte swelling could be abolished by mutating Lys 36 to alanine strongly suggests that the effect is hAQP1-dependent and that the described molecules bind at the extracellular entrance of the pore, close to the selectivity filter, and block water flux across the channel. The pivotal role of Lys36 for binding of compounds 1 and 2 renders molecules with this binding mode particularly interesting since it offers an attractive route for optimizing selectivity against other members of the hAQP family.

Conclusions. We present three novel hAQP1 inhibitors which block water flux across the cell membrane in the low micromolar range. Although no inhibitory effect was seen in a red blood cell assay, the predicted binding pose could be confirmed by a mutagenesis experiment, providing strong evidence that the dicovered molecules inhibit water flux by direct binding to hAQP1 at the extracellular site in close proximity to the ar/ $\mathrm{R}$ selectivity filter. The binding site is distinct from previously reported binding sites and is most likely suitable for the development of hAQP1-selective molecules as Lys36 is unique among the hAQP family. Although the presented molecules show only moderate efficacy in the low micromolar range, they have lead-like character and offer attractive entry points for the development of potent and selective hAQP1-blockers that are inevitable to further substantiate the various physiological roles of hAQP1 and its involvement in human pathologies and to elucidate the potential therapeutic benefit of hAQP1 inhibitors.

\section{EXPERIMENTAL SECTION}

Virtual Screening. Virtual screening was carried out by molecular docking of the lead-like subset $\left(\sim 10^{6}\right.$ compounds) of the ZINC (version 7) databas $^{37}$ to a structure that was taken from a $\mathrm{MD}$ simulation of hAQP1 with a quarternary ammonium inhibitor. ${ }^{32}$ As binding site a $12 \AA$ sphere around the inhibitor was defined that comprises the entire extracellular part of the pore including the A-loop of the neighboring monomer, which has been suggested to be involved in binding of quarternary ammonium compounds. Receptor and ligand preparation as well as docking was carried out with standard settings of the FlexX program. ${ }^{38}$ As we noticed that large compounds systematically scored higher than small ones, we did not use the FlexX docking scores for the final ranking but rather applied the ligand efficiency, which is defined as the overall score divided by the number of heavy atoms. This ensures that small compounds with favorable interactions appear higher in the ranked database.

Expression Constructs. Full-length human hAQP1 cDNA (a gift from Peter Deen, Radboud University Nijmegen Medical Centre, Netherlands) was cloned into the EcoRV/SpeI sites of the oocyte expression vector pT7Ts. $^{39}$ The K36A mutation into hAQP1 was introduced by using the QuickChange sitedirected mutagenesis kit (Stratagene) on pT7Ts-AQP1. Sequence analysis of selected clones was carried out to confirm that only the desired mutation was produced.

Functional Expression in Xenopus laevis Oocytes and Swelling Assays. Capped RNAs (cRNAs) were synthesized in vitro with T7 RNA Polymerase (Roche) from XbaI-linearized pT7Ts vector
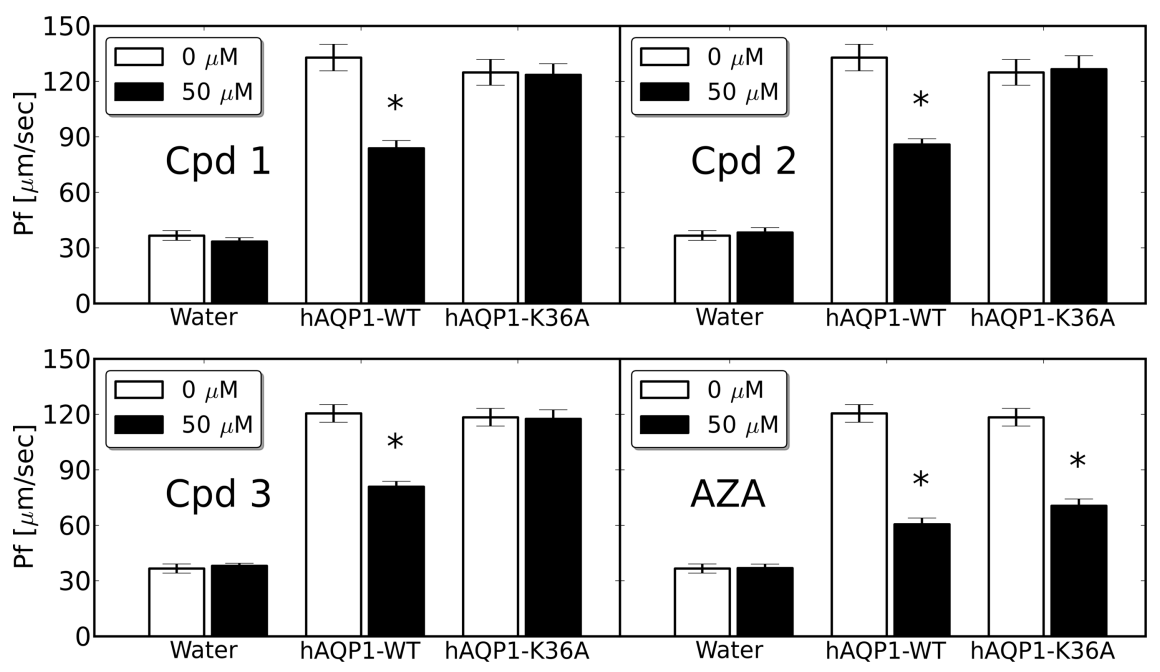

Figure 5. Inhibition of water flux in hAQP1-WT and hAQP1-K36A expressing X. leavis oocytes by three novel compounds (cpd1, cpd2, cpd3) and acetazolamide (AZA). Data (mean \pm SEM, $n=10-14$ oocytes) are the $P_{\mathrm{f}}$ of a representative experiment. Data show that all compounds inhibit water flux across hAQP1-WT, but only AZA inhibits water flux across the hAQP1-K36A mutant. *, $P<0.01$ vs oocytes not exposed to the compounds. 
containing hAQP1 or hAQP1-K36A. The isolation and microinjection of stage $\mathrm{V}$ oocytes was performed as described previously. ${ }^{39}$ Oocytes were injected with $50 \mathrm{~nL}$ of distilled water (negative control) or $50 \mathrm{~nL}$ of water solution containing 0.05 ng wild-type hAQP1 (hAQP1-WT) or hAQP1-K36A cRNAs. The osmotic water permeability $\left(P_{\mathrm{f}}\right)$ was measured $48 \mathrm{~h}$ after injection from the time course of osmotic oocyte swelling in a standard assay. Oocytes were transferred from $200 \mathrm{mOsm}$ modified Barth's culture medium (MBS; $0.33 \mathrm{mM} \mathrm{Ca}\left(\mathrm{NO}_{3}\right)_{2}, 0.4 \mathrm{mM}$ $\mathrm{CaCl}_{2}, 88 \mathrm{mM} \mathrm{NaCl}, 1 \mathrm{mM} \mathrm{KCl}, 2.4 \mathrm{mM} \mathrm{NaHCO}_{3}, 10 \mathrm{mM}$ Hepes, $\left.0.82 \mathrm{mM} \mathrm{MgSO}_{4}, \mathrm{pH} 7.5\right)$ to $20 \mathrm{mOsm} \mathrm{MBS}$ at RT. Oocyte swelling was followed by video microscopy using serial images at $2 \mathrm{~s}$ intervals during the first $20 \mathrm{~s}$ period. The $P_{\mathrm{f}}$ values were calculated taking into account the time-course changes in relative oocyte volume $\left[\mathrm{d}\left(V / V_{0}\right) /\right.$ $\mathrm{d} t]$, the molar volume of water $\left(V_{\mathrm{w}}=18 \mathrm{~cm}^{3} / \mathrm{mL}\right)$, and the oocyte surface area $(S)$ using the formula $V_{0}\left[\mathrm{~d}\left(V / V_{0}\right) / \mathrm{d} t\right] /\left[S V_{\mathrm{w}}\left(\mathrm{Osm}_{\mathrm{in}}-\right.\right.$ $\left.\left.\mathrm{Osm}_{\text {out }}\right)\right]$. For the initial screening of the compounds, water- and aquaporin-injected oocytes were preincubated with different concentrations of DMSO-dissolved drugs $(50,100$, and $300 \mu \mathrm{M})$ for $15 \mathrm{~min}$ in MBS before and during the swelling assays. The final concentration of DMSO in both treated and untreated oocytes was 1\%. For determination of the $\mathrm{IC}_{50}$ of $\mathrm{AZA}, \mathrm{cpd} 1, \mathrm{cpd} 2$, and cpd3 pairwise experiments were carried out. In these experiments, each oocyte served as its own control and was assayed first without drug treatment, incubated for $1-2 \mathrm{~h}$ in isotonic MBS, exposed to the drug (1-300 $\mu \mathrm{M})$ for $15 \mathrm{~min}$ and reassessed in a second swelling assay. To compile results from multiple batches and calculate the percentage block of $P_{\mathrm{f}}$ data were standardized as a percentage of the mean swelling rate of untreated hAQP-WT-expressing oocytes on the same day. Data were further subjected to the automatic curve-fitting procedure in SigmaPlot v.12.

Blood Cell Measurements. Blood drawn from the experimenter's fingertips was immediately suspended in ice-cold Carlsen buffer (154 $\mathrm{mM} \mathrm{NaCl}, 5 \mathrm{mM}$ D-glucose, $0.25 \mathrm{mM} \mathrm{KH} \mathrm{PO}_{4}, 0.25 \mathrm{mM} \mathrm{Na}_{2} \mathrm{HPO}_{4}$, $\mathrm{KOH} \mathrm{pH} \mathrm{7.4,} 300 \mathrm{mOsm})$ and washed three times $(2000 \mathrm{~g}, 277 \mathrm{~K}, 10$ $\min ) .^{40,41}$ For inhibition studies, the blood cell suspension (hematocrit $\left.\sim 7 \%, \mathrm{OD}_{600} 1 / 100 \sim 1.6\right)$ was diluted 20 -fold $\left(\mathrm{OD}_{600} \sim 8\right)$ in ice-cold Carlsen buffer containing only DMSO $(0.1 \% \mathrm{v} / \mathrm{v}$ final concentration) or the test compounds in addition at concentrations of $20 \mu \mathrm{M}$ for compounds $\mathbf{1}$ and 2 and $4 \mu \mathrm{M}$ for compound 3 due to its solubility in the assay buffer, followed by incubation (10-20 min, 273-299 K) inside a stopped-flow apparatus (SFM300, BioLogic, Claix, France). For comparability, compound $\mathbf{2}$ was tested in the presence of DMSO despite the stock solution being aqueous. Incubation with mercuric chloride was in the absence of DMSO and at lower blood cell density $\left(\mathrm{OD}_{600} \sim 2\right)$. Water and glycerol permeability measurements were performed at constant temperature $(299 \mathrm{~K})$ by following changes in light scattering $\left(90^{\circ}, \lambda=600 \mathrm{~nm}\right)$ after mixing preincubated blood cell suspension with an equal volume of glycerol-containing Carlsen buffer (60 mM glycerol, $30 \mathrm{mM}$ final gradient). Both buffers contained equal amounts of DMSO and test compound. Nine time courses $(8 \mathrm{~s})$ were recorded for each sample. Normalized average curves were used for evaluation.

Molecular Dynamics Simulations. Molecular dynamics simulations were carried out using the gromacs- 4.0 package. ${ }^{42,43}$ The simulation system of hAQP1 consists of the AQP tetramer embedded in DOPC lipid bilayer ${ }^{44}$ and solvated in water. $\mathrm{NaCl}$ was added to achieve a $150 \mathrm{mM}$ salt concentration. The amber99sb force field $\mathrm{d}^{45,46}$ and the TIP $3 \mathrm{P}^{47}$ water model was used. Ligand parameters were obtained according to the generalized amber force field (GAFF) procedure $^{48}$ with partial charges derived from quantum mechanical calculations with Gaussian03 ${ }^{49}$ (Hartree-Fock/6-31G* basis set). Amber topologies for the ligands were converted to gromacs format using the amb2gmx.pl script from Mobley et al. ${ }^{50}$ Simulations were started from the docked ligand configurations. After energy minimization and $50 \mathrm{ps}$ of equilibration with positional restraints on heavy atoms, trajectories of $100 \mathrm{~ns}$ length for each of the three compounds were collected at $300 \mathrm{~K}$ with standard NPT ensemble settings (thermostat: velocity-rescaling; ${ }^{51,52}$ barostat: Parinello-Rah$\left.\operatorname{man}^{53}\right)$. Electrostatic interactions were calculated at every step with the particle-mesh Ewald method, ${ }^{54}$ and short-range repulsive and attractive dispersion interactions were simultaneously described by a Lennard-Jones potential, which was cut off at $1.0 \mathrm{~nm}$. The SETTLE ${ }^{55}$ algorithm was used to constrain bonds and angles of water molecules, and LINCS $^{56}$ was used for all other bonds, allowing a time step of $2 \mathrm{fs}$.

Free-Energy Calculations. The effect of the K36A mutation in ligand binding was assessed using the Crooks-Gaussian-Intersection protocol. ${ }^{57}$ Simulation topologies and input files were generated using a previously described procedure for the setup of hybrid amino acid topologies. ${ }^{35}$ For each state $(\lambda=0$ and $\lambda=1$, representing Lys and Ala, respectively) three independent simulations of $20 \mathrm{~ns}$ length each for both apo-hAQP1 and ligand-bound hAQP1 were conducted using the simulation parameters of ref 35 . From each trajectory, snapshots were taken every $160 \mathrm{ps}$, and short nonequilibrium thermodynamic integration runs (100 ps) were performed in which $\lambda$ was switched from 0 to 1 or from 1 to 0 , respectively. The derivative of the Hamiltonian with respect to $\lambda$ was recorded at every step and the alchemical free energy for the transition was calculated according to Goette and Grubmueller. ${ }^{57}$ The binding free energy difference was calculate according to $\Delta \Delta G_{\text {bind }}=\Delta G_{\text {cmplx }}-\Delta G_{\text {apo }}$. The results are shown in the Supporting Material.

Statistics. Data are presented as mean \pm SEM. The $P_{\mathrm{f}}$ data of hAQP1-WT and hAQP1-K36A-expressing oocytes, in the presence or absence of the inhibitory compounds, was statistically analyzed by the Student's $t$ test. $P$ value of $<0.05$ was considered statistically significant.

\section{ASSOCIATED CONTENT}

Supporting Information

This material is available free of charge via the Internet at http://pubs.acs.org.

\section{AUTHOR INFORMATION}

\section{Corresponding Author}

*E-mail: joan.cerda@irta.cat; bgroot@gwdg.de.

\section{Present Address}

"Boehringer Ingelheim Pharma.

\section{Author Contributions}

${ }^{\perp}$ These authors contributed equally to this work.

Notes

The authors declare no competing financial interest.

\section{ACKNOWLEDGMENTS}

The authors thank C. Aponte-Santamaria for help with the calculation of permeation rates and M. Rutzler (University of Aarhus) for initial experiments. Funding by the EC, contract HEALTH-F4-2007-201924 (EDICT), and the Deutsche Forschungsgemeinschaft (DFG), grant No. GR 207914, is gratefully acknowledged.

\section{REFERENCES}

(1) de Groot, B. L., and Grubmüller, H. (2001) Water permeation across biological membranes: Mechanism and dynamics of Aquaporin1 and GlpF. Science 294, 2353-2357.

(2) Hub, J., and de Groot, B. (2008) Mechanism of selectivity in aquaporins and aquaglyceroporins. Proc. Natl. Acad. Sci. U.S.A. 105, $1198-1203$.

(3) Preston, G. M., Carroll, T. P., Guggino, W. B., and Agre, P. (1992) Appearance of water channels in Xenopus oocytes expressing red-cell CHIP28 protein. Science 256, 385-387.

(4) Zeidel, M. L., Ambudkar, S. V., Smith, B. L., and Agre, P. (1992) Reconstitution of functional water channels in liposomes containing purified red-cell CHIP28 protein. Biochemistry 31, 7436-7440.

(5) Fujiyoshi, Y., Mitsuoka, K., de Groot, B. L., Philippsen, A., Grubmüller, H., Agre, P., and Engel, A. (2002) Structure and function of water channels. Curr. Opin. Struct. Biol. 12, 509-515.

(6) Hoque, M., Soria, J., Woo, J., Lee, T., Lee, J., Jang, S., Upadhyay, S., Trink, B., Monitto, C., Desmaze, C., Mao, L., Sidransky, D., and 
Moon, C. (2006) Aquaporin 1 is overexpressed in lung cancer and stimulates NIH-3T3 cell proliferation and anchorage-independent growth. Am. J. Pathol. 168, 1345-1353.

(7) King, L. S., Kozono, D., and Agre, P. (2004) From structure to disease: the evolving tale of aquaporin biology. Nat. Rev. Mol. Cell Biol. $5,687-698$.

(8) Verkman, A. (2005) More than just water channels: unexpected cellular roles of aquaporins. J. Cell. Sci. 118, 3225-3232.

(9) Saadoun, S., Papadopoulos, M., Davies, D., Bell, B., and Krishna, S. (2002) Increased aquaporin 1 water channel expression in human brain tumours. Br. J. Cancer 87, 621-623.

(10) Frigeri, A., Gropper, M., Turck, C., and Verkman, A. (1995) Immunolocalization of the mercurial-insensitive water channel and glycerol intrinsic protein in epithelial cell plasma membranes. Proc. Natl. Acad. Sci. U.S.A. 92, 4328-4331.

(11) Zhang, D., Vetrivel, L., and Verkman, A. (2002) Aquaporin deletion in mice reduces intraocular pressure and aqueous fluid production. J. Gen. Physiol. 119, 561-569.

(12) Nielsen, S., Smith, B., Christensen, E., and Agre, P. (1993) Distribution of the aquaporin CHIP in secretory and resorptive epithelia and capillary endothelia. Proc. Natl. Acad. Sci. U.S.A. 90, 7275-7279.

(13) Oshio, K., Watanabe, H., Song, Y., Verkman, A., and Manley, G. (2005) Reduced cerebrospinal fluid production and intracranial pressure in mice lacking choroid plexus water channel Aquaporin-1. FASEB J. 19, 76-78.

(14) Oshio, K., Song, Y., Verkman, A., and Manley, G. (2003) Aquaporin-1 deletion reduces osmotic water permeability and cerebrospinal fluid production. Acta Neurochir. Suppl. 86, 525-528.

(15) Rodríguez, A., Pérez-Gracia, E., Espinosa, J., Pumarola, M., Torres, J., and Ferrer, I. (2006) Increased expression of water channel aquaporin 1 and aquaporin 4 in Creutzfeldt-Jakob disease and in bovine spongiform encephalopathy-infected bovine-PrP transgenic mice. Acta Neuropathol. 112, 573-585.

(16) $\mathrm{Hu}, \mathrm{J}$., and Verkman, A. (2006) Increased migration and metastatic potential of tumor cells expressing aquaporin water channels. FASEB J. 20, 1892-1894.

(17) Verkman, A., Hara-Chikuma, M., and Papadopoulos, M. (2008) Aquaporins-new players in cancer biology. J. Mol. Med. 86, 523-529.

(18) Saadoun, S., Papadopoulos, M., Hara-Chikuma, M., and Verkman, A. (2005) Impairment of angiogenesis and cell migration by targeted aquaporin-1 gene disruption. Nature 434, 786-792.

(19) Vacca, A., Ribatti, D., Roccaro, A., Frigeri, A., and Dammacco, F. (2001) Bone marrow angiogenesis in patients with active multiple myeloma. Semin. Oncol. 28, 543-550.

(20) Vacca, A., Frigeri, A., Ribatti, D., Nicchia, G., Nico, B., Ria, R., Svelto, M., and Dammacco, F. (2001) Microvessel overexpression of aquaporin 1 parallels bone marrow angiogenesis in patients with active multiple myeloma. Br. J. Hamaetol. 113, 415-421.

(21) Zhang, H., and Verkman, A. (2009) Aquaporin-1 tunes pain perception by interaction with $\mathrm{Na}_{\nu} 1.8 \mathrm{Na}^{+}$channels in dorsal root ganglion neurons. J. Biol. Chem. 285, 5896-5906.

(22) Yool, A. (2007) Aquaporins: multiple roles in the central nervous system. Neuroscientist 13, 470-485.

(23) Frigeri, A., Nicchia, G., and Svelto, M. (2007) Aquaporins as targets for drug discovery. Curr. Pharm. Des. 13, 2421-2427.

(24) Preston, G. M., Jung, J. S., Guggino, W. B., and Agre, P. (1993) The mercury-sensitive residue at cysteine-189 in the CHIP28 water channel. J. Biol. Chem. 268, 17-20.

(25) Detmers, F., de Groot, B., Muller, E., Hinton, A., Konings, I., Sze, M., Flitsch, S., Grubmuller, H., and Deen, P. (2006) Quaternary ammonium compounds as water channel blockers: specificity, potency, and site of action. J. Biol. Chem. 281, 14207-14214.

(26) Yool, A., Brokl, O., Pannabecker, T., Dantzler, W., and Stamer, W. (2002) Tetraethylammonium block of water flux in Aquaporin-1 channels expressed in kidney thin limbs of Henle's loop and a kidneyderived cell line. BMC physiol. 2, 1-8.
(27) Brooks, H., Regan, J., and Yool, A. (2000) Inhibition of aquaporin-1 water permeability by tetraethylammonium: involvement of the loop E pore region. Mol. Pharmacol. 57, 1021-1026.

(28) Niemietz, C., and Tyerman, S. (2002) New potent inhibitors of aquaporins: silver and gold compounds inhibit aquaporins of plant and human origin. FEBS Lett. 531, 443-447.

(29) Bing, M., Xiang, Y., Sheng-mei, M., Tao, L., He-ming, Y., and Xue-jun, L. (2004) Effects of acetazolamide and anordiol on osmotic water permeability in AQP1-cRNA injected Xenopus oocyte. Acta Pharmacol. Sin. 25, 90-97.

(30) Migliati, E., Meurice, N., DuBois, P., Fang, J., Somasekharan, S., Beckett, E., Flynn, G., and Yool, A. (2009) Inhibition of Aquaporin-1 and Aquaporin-4 water permeability by a derivative of the loop diuretic bumetanide acting at an internal pore-occluding binding site. Mol. Pharmacol. 76, 105-112.

(31) Yang, B., Kim, J., and Verkman, A. (2006) Comparative efficacy of $\mathrm{HgCl} 2$ with candidate aquaporin-1 inhibitors DMSO, gold, TEA+ and acetazolamide. FEBS Lett. 580, 6679-6684.

(32) Müller, E., Hub, J., Grubmüller, H., and de Groot, B. (2008) Is TEA an inhibitor for human Aquaporin-1? Pflügers Arch. 456, 663669.

(33) Flower, D. (1998) On the properties of bit string-based measures of chemical similarity. J. Chem. Inf. Comput. Sci. 38, 379-386.

(34) Willett, P., Barnard, J., and Downs, G. (1998) Chemical similarity searching. J. Chem. Inf. Comput. Sci. 38, 983-996.

(35) Seeliger, D., and de Groot, B. (2010) Protein thermostability calculations using alchemical free energy simulations. Biophys. J. 98, 2309-2316.

(36) Seeliger, D., Buelens, F., Goette, M., de Groot, B., and Grubmüller, H. (2011) Towards computional specificity screening of DNA-binding proteins. Nucleic Acids Res. 39, 8281-8290.

(37) Irwin, J. J., and Shoichet, B. K. (2005) ZINC- A free database of commercially available compounds for virtual screening. J. Chem. Inf. Model. 45, 177-182.

(38) Rarey, M., Kramer, B., Lengauer, T., and Klebe, G. (1996) A fast docking method using an incremental construction algorithm. J. Mol. Biol. 261, 470-489.

(39) Deen, P., Verdijk, M., Knoers, N., Wieringa, B., Monnens, L., Van Os, C., and Van Oost, B. (1994) Requirement of human renal water channel aquaporin-2 for vasopressin-dependent concentration of urine. Science 264, 92-95.

(40) Carlsen, A., and Wieth, J. (1976) Glycerol transport in human red cells. Acta Physiol. Scand. 97, 501-513.

(41) Roudier, N., Verbavatz, J., Maurel, C., Ripoche, P., and Tacnet, F. (1998) Evidence for the presence of aquaporin-3 in human red blood cells. J. Biol. Chem. 273, 8407-8412.

(42) Van der Spoel, D.; Berendsen, H. J. C.; Van Buuren, A. R.; Apol, E.; Meulenhoff, P. J.; Sijbers, A. L. T. M.; Van Drunen, R. Gromacs User Manual; Royal Institute of Technology and Uppsala University: Sweden, 1995.

(43) Hess, B., Kutzner, C., van der Spoel, D., and Lindahl, E. (2008) GROMACS 4: Algorithms for highly efficient, load-balanced, and scalable molecular simulation. J. Chem. Theory Comput. 4, 435-447.

(44) Siu, S., Vácha, R., Jungwirth, P., and Böckmann, R. (2008) Biomolecular simulations of membranes: Physical properties from different force fields. J. Chem. Phys. 128, 125103-125112.

(45) Hornak, V., Abel, R., Okur, A., Strockbine, B., Roitberg, A., and Simmerling, C. (2006) Comparison of multiple Amber force fields and development of improved protein backbone parameters. Proteins: Struct. Funct. Bioinf. 65, 712-725.

(46) Sorin, E., and Pande, V. (2005) Exploring the helix-coil transition via all-atom equilibrium ensemble simulations. Biophys. J. 88, $2472-2493$

(47) Jorgensen, W. L., Chandrasekhar, J., Madura, J. D., Impey, R. W., and Klein, M. L. (1983) Comparison of simple potential functions for simulating liquid water. J. Chem. Phys. 79, 926-935.

(48) Wang, J., Wolf, R. M., Caldwell, J. W., Kollmann, P. A., and Case, D. A. (2004) Development and testing of a generalized amber force field. J. Comput. Chem. 25, 1157-1174. 
(49) Frisch, M. J.; Trucks, G. W.; Schlegel, H. B.; Scuseria, G. E.; Robb, M. A.; Cheeseman, J. R. A.; Montgomery, Jr., J.; Vreven, T.; Kudin, K. N.; Burant, J. C.; Millam, J. M.; Iyengar, S. S.; Tomasi, J.; Barone, V.; Mennucci, B.; Cossi, M.; Scalmani, G.; Rega, N.; Petersson, G. A.; Nakatsuji, H.; Hada, M.; Ehara, M.; Toyota, K.; Fukuda, R.; Hasegawa, J.; Ishida, M.; Nakajima, T.; Honda, Y.; Kitao, O.; Nakai, H.; Klene, M.; Li, X.; Knox, J. E.; Hratchian, H. P.; Cross, J. B.; Bakken, V.; Adamo, C.; Jaramillo, J.; Gomperts, R.; Stratmann, R. E.; Yazyev, O.; Austin, A. J.; Cammi, R.; Pomelli, C.; Ochterski, J. W.; Ayala, P. Y.; Morokuma, K.; Voth, G. A.; Salvador, P.; Dannenberg, J. J.; Zakrzewski, V. G.; Dapprich, S.; Daniels, A. D.; Strain, M. C.; Farkas, O.; Malick, D. K.; Rabuck, A. D.; Raghavachari, K.; Foresman, J. B.; Ortiz, J. V.; Cui, Q.; Baboul, A. G.; Clifford, S.; Cioslowski, J.; Stefanov, B. B.; Liu, G.; Liashenko, A.; Piskorz, P.; Komaromi, I.; Martin, R. L.; Fox, D. J.; Keith, T.; Al-Laham, M. A.; Peng, C. Y.; Nanayakkara, A.; Challacombe, M.; Gill, P. M. W.; Johnson, B.; Chen, W.; Wong, M. W.; Gonzalez, C.; Pople, J. A. Gaussian 03, Revision C.02; Gaussian, Inc.: Wallingford, CT, 2004.

(50) Mobley, D., Chodera, J., and Dill, K. (2006) On the use of orientational restraints and symmetry corrections in alchemical free energy calculations. J. Chem. Phys. 125, 084902-084902.

(51) Berendsen, H. J. C., Postma, J. P. M., DiNola, A., and Haak, J. R. (1984) Molecular dynamics with coupling to an external bath. J. Chem. Phys. 81, 3684-3690.

(52) Bussi, G., Donadio, D., and Parrinello, M. (2007) Canonical sampling through velocity rescaling. J. Chem. Phys. 126, 014101-1014101-7.

(53) Parrinello, M., and Rahman, A. (1981) Polymorphic transitions in single crystals: A new molecular dynamics method. J. Appl. Phys. 52, $7182-7190$.

(54) Essmann, U., Perera, L., Berkowitz, M. L., Darden, T., Lee, H., and Pedersen, L. G. (1995) A smooth particle mesh ewald potential. J. Chem. Phys. 103, 8577-8592.

(55) Miyamoto, S., and Kollman, P. A. (1992) SETTLE: An analytical version of the SHAKE and RATTLE algorithms for rigid water models. J. Comput. Chem. 13, 952-962.

(56) Hess, B., Bekker, H., Berendsen, H. J. C., and Fraaije, J. G. E. M. (1997) LINCS: A linear constraint solver for molecular simulations. J. Comput. Chem. 18, 1463-1472.

(57) Goette, M., and Grubmüller, H. (2008) Accuracy and convergence of free energy differences calculated from nonequilibrium switching processes. J. Comput. Chem. 30, 447-456. 